\title{
The role of PDIA3 in myogenesis during muscle regeneration
}

\author{
Chao Wang ${ }^{1,2}$, Yuanjiao Zhu', Dan Wu' ${ }^{1}$, Zien Wang ${ }^{3}$, Xiaoli Xu ${ }^{4}$, Yan Shi ${ }^{1}$, Gang Yang ${ }^{1}$, Yongming Yu ${ }^{5}$ and Xi Peng ${ }^{1,3,5}$
}

\begin{abstract}
Beta 3 ( $\beta 3$ ) integrin plays an important role in the initiation of myogenesis in adult muscle. Protein disulfide isomerases (PDIs) can activate $\beta 3$ integrin in various cells to promote cell migration, adhesion and fusion. However, the effect of PDIs on myogenesis during muscle regeneration has not been elucidated. Here, we report that PDIA3 expression is induced in regenerating myofibers. The inhibition of PDIA3 in muscle injuries in mice disrupts myoblast differentiation, impairs muscle regeneration, and ultimately aggravates muscle damage. Moreover, PDIA3 expression is upregulated and observed on the cell surfaces of myoblasts during differentiation and fusion. The inhibition of extracellular PDIA3 with an anti-PDIA3 monoclonal antibody attenuates $\beta 3$ integrin/AKT/mTOR signal activity, inhibits myoblast differentiation, and blocks the fusion of myoblasts. Thus, PDIA3 may be a mediator of myoblast differentiation and fusion during muscle regeneration.
\end{abstract}

\section{Introduction}

Skeletal muscle is the major component of the human locomotion system. It is also recognized as a metabolic organ that supplies amino acids and utilizes glucose and fatty acids ${ }^{1-3}$. Skeletal muscle damage is a frequent clinical problem that is usually secondary to burn injury, trauma, and myopathy caused by conditions such as Duchenne muscular dystrophy (DMD $)^{4-8}$. Muscle regeneration deficiency causes injuries to heal slowly and improperly, resulting in muscle fibrosis and the loss of normal muscle function ${ }^{9,10}$. In contrast, efficient muscle regeneration following muscle injury is crucial for restoring the physiological function of skeletal muscle. Therefore, skeletal muscle regeneration is an essential process required for maintaining the contractile function of muscle and whole-body energy metabolism ${ }^{10,11}$.

The regenerative capacity of skeletal muscle mainly depends on satellite cells (SCs), which are normally

\footnotetext{
Correspondence: Xi Peng (pxIrmm@163.com)

${ }^{1}$ State Key Laboratory of Trauma, Burns and Combined Injury, Clinical Medical Research Center, Southwest Hospital, Third Military Medical University (The Army Medical University), Chongqing 400038, China

2Department of Burns and Plastic Surgery, Chengdu Second People's Hospital, Chengdu 610011, China
}

Full list of author information is available at the end of the article. quiescent. After muscle injury, these cells are induced to undergo myogenesis through coordinated myogenic marker expression and cell migration and fusion and ultimately form new myofibers ${ }^{12,13}$. However, the mechanisms by which SCs sense muscle damage and undergo myogenesis in an orderly manner have not been fully elucidated.

Integrins are a family of transmembrane receptors that mediate cell-cell and cell-extracellular matrix (ECM) adhesion $^{14}$. In addition, integrins can cooperate with multiple growth factors and generate many intracellular signals to regulate cell proliferation, differentiation, and fusion. Integrins are heterodimers of two subunits, $\alpha$ and $\beta$. Integrins in mammals have $24 \alpha$ and $9 \beta$ subunits $^{15-17}$. In skeletal muscle, the integrin $\alpha \mathrm{V} \beta 3$ is crucial for the maintenance of muscle integrity, as mice with defects in $\beta 3$ integrin exhibit the failure of myogenesis during muscle regeneration. In addition, $\alpha \mathrm{V} \beta 3$ integrin has been demonstrated to mediate myogenesis through promoting myogenic gene expression and the migration and fusion of $\mathrm{SCs}^{18}$.

Protein disulfide isomerases (PDIs) are multifunctional chaperones that regulate multiple protein functions via the oxidation, reduction, and isomerization of protein 
disulfide bonds. Most PDI family members (PDIs) are localized within the endoplasmic reticulum (ER); however, some PDI family members can escape from the ER and be secreted into the ECM or translocate to the cell surface, such as PDIA1 and PDIA $3^{19}$.

Extracellular PDIA1 and PDIA3 have recently been identified as two activators of $\alpha \mathrm{V} \beta 3$ integrin through isomerization of disulfide bonds in integrin $\beta 3$ subunits. The interaction between PDI and $\beta 3$ integrin plays a key role in mediating multiple biological processes, including platelet adhesion ${ }^{20}$, neutrophil migration ${ }^{21}, \mathrm{~T}$ cell migration $^{22}$, and gamete fusion ${ }^{23}$. Blockade of either PDIA1 or PDIA3 at the cell surface causes $\beta 3$ integrin inactivation and then impairs the cellular capacities for migration, adhesion, and fusion, which are all basic cell behaviors involved in myogenesis during muscle regeneration ${ }^{18,20-22}$. However, whether the mediation of the activation of $\beta 3$ integrin by PDIA1 and PDIA3 plays a role in myogenesis is unknown.

Here, we hypothesized that PDI family members play a major regulatory role in myogenesis through their interactions with $\beta 3$ integrin. In the present study, we revealed for the first time that PDIA3 is persistently expressed in neonatal myofibers during muscle regeneration and that it facilitates myogenesis via mediating myogenic differentiation and fusion.

\section{Materials and methods \\ Reagents}

Antibodies against eMyHC, myogenin, Pax7, and $\beta 3$ integrin were purchased from Santa Cruz (Dallas, TX, USA). Antibodies against AKT, p-AKT, Myod1, PDIA1, PDIA3, and GAPDH were purchased from Abcam (Cambridge, UK). Antibodies against mTOR and PmTOR were purchased from Cell Signaling Technology (Beverly, MA, USA). Antibodies against $\mathrm{eMyHC}$ were purchased from R\&D (Minneapolis, MN, USA). The Cell Surface Protein Isolation Kit, Donkey anti-rabbit FITC, and donkey anti-mouse TRITC were purchased from Thermo Fisher Scientific (Waltham, MA USA). The ELISA kit for PDIA3 detection was obtained from R\&D (Minneapolis, MN, USA). Cardiotoxin (CTX), LY294002, recombinant human Vitronectin and PDI inhibitors (16F16, EGCG, bacitracin, and PCAMA31) were obtained from Sigma-Aldrich (St. Louis, MO, USA).

\section{Animal model}

Male C57 mice (6 weeks old, 20-25 g) were purchased from the Third Military Medical University Laboratory Animal Center. A total of $100 \mu \mathrm{l} \mathrm{CTX}(10 \mu \mathrm{M}$, dissolved in PBS) was injected into the gastrocnemius of mice to generate a model of muscle injury. At 3 and 7 days postinjury, the gastrocnemius was excised from euthanized mice to conduct the described analyses. In our study, all animal experimental protocols were approved by the Animal Care Committee of the Third Military Medical University according to the National Institutes of Health Guide for the Care and Use of Laboratory Animals (NIH publication number 8023, revised 1978).

\section{Myogenic cell culture}

Murine $\mathrm{C} 2 \mathrm{C} 12$ muscle cells were purchased from ATCC (Manassas, USA) and grown in high-glucose DMEM supplemented with $10 \%$ fetal bovine serum (HyClone, USA) and $1 \%$ penicillin/streptomycin at $37^{\circ} \mathrm{C}$ in $5 \% \mathrm{CO}_{2}$. Once they reached $70 \%$ confluence, $\mathrm{C} 2 \mathrm{C} 12$ myoblasts were induced to differentiate for $48 \mathrm{~h}$ by adding DMEM containing $2 \%$ horse serum.

\section{Cell surface protein extraction}

The Thermo Scientific Pierce Cell Surface Protein Isolation Kit (89881) enables the convenient biotinylation and isolation of cell surface proteins for Western blot analysis. Cells are first labeled with Thermo Scientific EZLink Sulfo-NHS-SS-Biotin, a thiol-cleavable amine-reactive biotinylation reagent. Cells are subsequently lysed with a mild detergent, and the labeled proteins are then isolated with Thermo Scientific NeutrAvidin Agarose. The bound proteins are released by incubating the lysates with SDS-PAGE sample buffer containing $50 \mathrm{mM}$ DTT.

\section{Immunoblotting and coimmunoprecipitation assays}

Immunoblotting assays were performed as described previously (4). Briefly, the proteins extracted from muscle and $\mathrm{C} 2 \mathrm{C} 12$ cells were separated by SDS-PAGE and transferred to PVDF membranes. These membranes were incubated at $4{ }^{\circ} \mathrm{C}$ overnight with anti-PDIA1 (1:500), antiPDIA3 (1:500), anti-eMyHC (1:200), anti-myogenin (1:1000), anti-Pax7 (1:1000), anti-Myod1 (1:1000) antieMyHC (1:500), anti-AKT (1:2000), anti-p-AKT (1:2000), anti-mTOR (1:500), anti-p-mTOR (1:500), anti- $\beta 3$ integrin (1:500), and GAPDH (1:2500) antibodies, followed by three washes with TBST and incubation with the associated secondary antibodies at room temperature (RT) for $60 \mathrm{~min}$. Following washing with TBST three times, the proteins of interest were detected with ECL reagents. The total proteins were immunoprecipitated with an antiPDIA3 antibody and then immunoblotted with an anti- $\beta 3$ integrin antibody.

\section{q-PCR}

RNA was isolated from muscle and $\mathrm{C} 2 \mathrm{C} 12$ cells using TRIzol (Invitrogen, USA). This RNA was used as a template for cDNA synthesis using reverse transcriptase (Invitrogen, USA). qPCR was performed with a sequence detection system (Bio-Rad, USA) with the following primers (Invitrogen, USA): eMyHC (forward, reverse) primers $5^{\prime}$-TCAGGATTCGGAGGAGCAGG-3' and $5^{\prime}$-C 
TTCTTGTCCAGAGCGGCAG-3'; myogenin (forward, reverse) primers 5'-GGTCCCAACCCAGGAGATCA-3' and $5^{\prime}$-CGTCTGGGAAGGCAACAGAC-3'; Myod1 (forward, reverse) primers $5^{\prime}$-ATGGCTACGACACC GCCTAC-3' and 5'-AGATGCGCTCCACTATGCTG-3'; PDIA1 (forward, reverse) primers 5'-CTGGCAG CAGAGGCTATTGA-3' and $5^{\prime}$-AAGTTGTTGCGGCC TTCATC-3'; PDIA3 (forward, reverse) primers $5^{\prime}$-ATG GATGCCACAGCCAATG- $3^{\prime}$ and $5^{\prime}$-TCACGGC CACCTTCATACTTC-3'; GAPDH (forward, reverse) primers $5^{\prime}$-CTGCGACCACCAACTGCTTAGC-3' and 5'-CTTCACCACCTTCTTGATGTC-3'.

\section{Immunofluorescence}

Frozen sections of excised gastrocnemius tissue were fixed with acetone at $4{ }^{\circ} \mathrm{C}$ for $10 \mathrm{~min}$, permeated with $0.03 \%$ Triton X-100 at RT for $30 \mathrm{~min}$, and blocked with $2 \%$ BSA in PBS at RT for $60 \mathrm{~min}$. Immunofluorescence staining of the muscle sections was carried out using primary antibodies against Pax7, myogenin, eMyHC, PDIA3, P-AKT, and $\beta 3$ integrin at $4{ }^{\circ} \mathrm{C}$ overnight, and then the sections were incubated with the associated secondary antibodies (donkey anti-mouse TRITC or donkey anti-rabbit FITC) at RT for $1 \mathrm{~h}$. Cells were seeded on rat tail collagen I (Invitrogen, USA)-coated coverslips for the differentiation analysis. At 1 and 2 days after differentiation, $\mathrm{C} 2 \mathrm{C} 12$ cells were fixed with $4 \%$ polyoxymethylene at RT for 15 min and blocked with $2 \%$ BSA at RT for $30 \mathrm{~min}$. The $\mathrm{C} 2 \mathrm{C} 12$ cells were then incubated with primary antibodies against PDIA3 $(1: 1000)$ at $4{ }^{\circ} \mathrm{C}$ for $1 \mathrm{~h}$, followed by incubation with the associated secondary antibodies for $1 \mathrm{~h}$ at $37^{\circ} \mathrm{C}$. The cell nuclei in muscle and $\mathrm{C} 2 \mathrm{C} 12$ cells were labeled by DAPI (blue). All images were acquired and analyzed using a Zeiss LSM 720 confocal microscope.

\section{Histology}

Paraffin-embedded and H\&E-stained gastrocnemius sections were generated by the Third Military Medical University Biomedical Analysis Center.

\section{Statistical analysis}

All experimental data are presented as the mean \pm standard error of the mean. The statistical analysis of these data was assessed for significance using Student's $t$ test and one-way analysis of variance. The results with $P$ values of $<0.05$ were considered to be significant. All data were analyzed with SPSS software (version 17.0).

\section{Results}

Upregulation of PDI in regenerated skeletal muscle

To test whether PDI participated in the regulation of skeletal muscle regeneration, we first measured the mRNA and protein levels of PDIA1 and PDIA3 in regenerated adult mouse skeletal muscle in the CTX injury model. As illustrated in Fig. 1a, the level of PDIA1 mRNA was increased on day 3 postinjury and dramatically downregulated to a normal level on day 7 postinjury. PDIA1 protein was expressed in uninjured muscles and was not altered at 3 days and 7 days after injury (Fig. 1g). In contrast, the level of PDIA3 mRNA was significantly increased on days 3 and 7 postinjury (Fig. 1b). Although PDIA3 was hardly detected in normal muscles, a significant increase in this protein was observed at 3 days and 7 days after injury (Fig. 1g). The immunofluorescence staining also showed that PDIA3 was induced in regenerated myofibers with centralized nuclei (Fig. 1h). It was notable that PDIA3 expression was upregulated and accompanied by the expression of myogenic markers, such as Pax7, Myod1, myogenin, and embryonic MyHC (eMyHC) (Fig. 1c-g). To further elucidate the role and mechanism of PDIA3 in myogenesis during muscle regeneration, immunofluorescence staining of PDIA3 and myogenic protein markers were simultaneously performed on frozen sections of skeletal muscles after injury induced by CTX. PDIA3 was hardly observed in activated SCs that were stained by Pax7 and Myod1 (Fig. 2) but was detected in neonatal myofibers that were myogenin- and eMyHC-positive (Fig. 2). These results suggest that PDIA3 plays a major role in terminal myogenesis during muscle regeneration.

\section{Inhibition of PDI activity attenuates muscle regeneration}

To evaluate the role of PDIA3 in muscle regeneration in vivo, we tested whether the inhibition of PDIA3 impaired myogenesis and muscle regeneration in mice. At 3 days after CTX-induced injury, when myogenesis occurred, the expression of Myod1, myogenin, and eMyHC were decreased after administration of a PDI inhibitor (PCAMA31) (Fig. 3a, b). However, Pax7 expression was increased in PCAMA31-treated mice with injured muscles (Fig. 3a). In addition, the inhibition of PDIA3 by EGCG significantly increased Pax7 expression, and decreased myogenin and eMyHC expressions but had no effect on Myod1 expression (Fig. 3c, d). As shown in Fig. 3e, the amount of eMyHC expressed in regenerated myofibers was significantly reduced in both PCAMA31treated and EGCG-treated mice compared with that in control muscles. Thus, PDIA3 appears to be essential for myogenic terminal differentiation. Finally, on day 14 after injury, regenerated myofibers with centralized nuclei predominated muscles in control mice but not those in PCAMA31-treated and EGCG-treated mice (Fig. 3f). Moreover, in treated mice, a pathologically high level of local inflammation was present, indicating the impairment of skeletal muscle regeneration (Fig. 3f). These data suggest that PDIA3 is critical to terminal differentiation during skeletal muscle regeneration. 


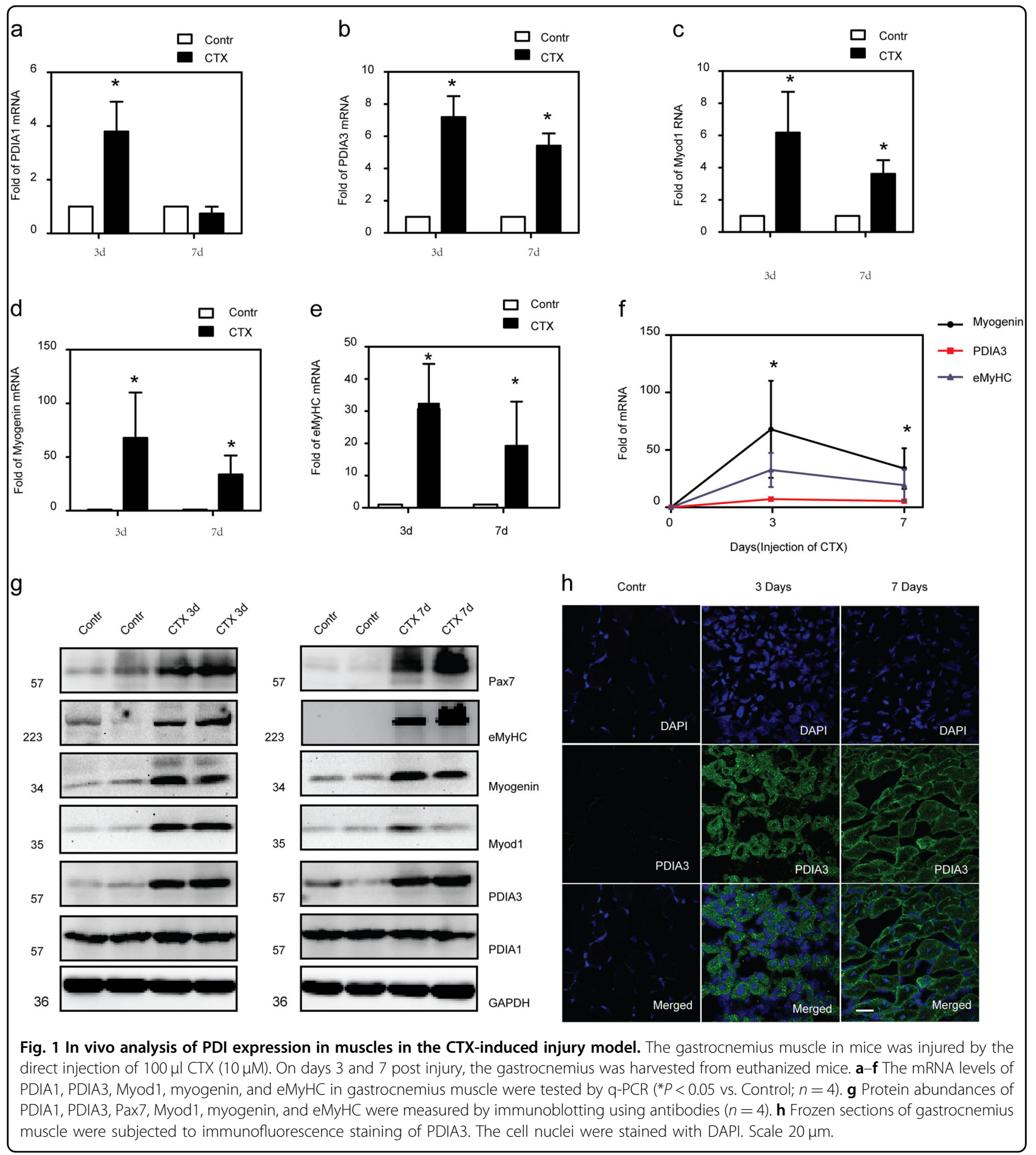

The role of PDIA3 in myoblast differentiation and fusion

To definitively confirm that PDIA3 functions in myogenesis, we employed multiple in vitro differentiation assays using $\mathrm{C} 2 \mathrm{C} 12$ myoblasts, which are most frequently used to study the mechanisms of myogenic differentiation. Immunoblotting showed that both PDIA1 and PDIA3 were expressed in undifferentiated myoblasts and that PDIA3 expression was correlated with the levels of the myogenic marker proteins myogenin and $\mathrm{MyHC}$, which increased gradually during $\mathrm{C} 2 \mathrm{C} 12$ myoblast differentiation. However, the expression of PDIA1 was unaltered (Fig. 4a).

To further test whether PDIA3 mediated myoblast differentiation, we inhibited PDI activity by utilizing PDI 


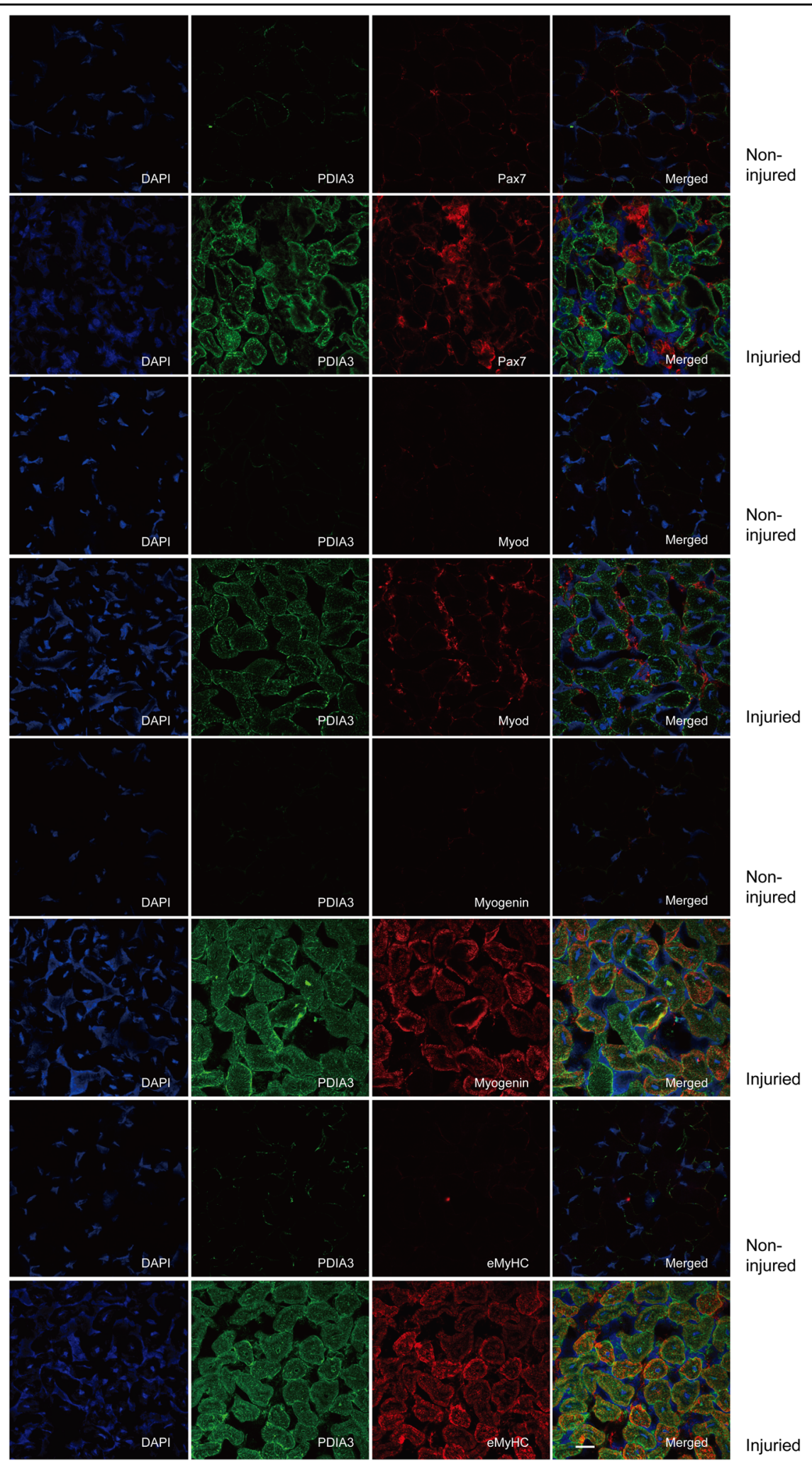

Fig. 2 Expression of PDIA3 in activated SCs during muscle regeneration. The gastrocnemius muscle in mice was injured by the direct injection of $100 \mu \mathrm{l} \mathrm{CTX}(10 \mu \mathrm{M})$. On day 7 post injury, the gastrocnemius muscle was harvested from euthanized mice. Frozen sections of gastrocnemius muscle were subjected to the immunofluorescence costaining of PDIA3 with Pax7, Myod1, myogenin, and eMyHC. The staining of Pax7 was performed to identify SCS; the staining of Myod 1 was used to identify activated SCs that had undergone differentiation. The staining of myogenin was performed to identify mature myotubes. The staining of eMyHC was used to identify regenerated myofibers. The cell nuclei were stained with DAPI. Scale $20 \mu \mathrm{m}$. 


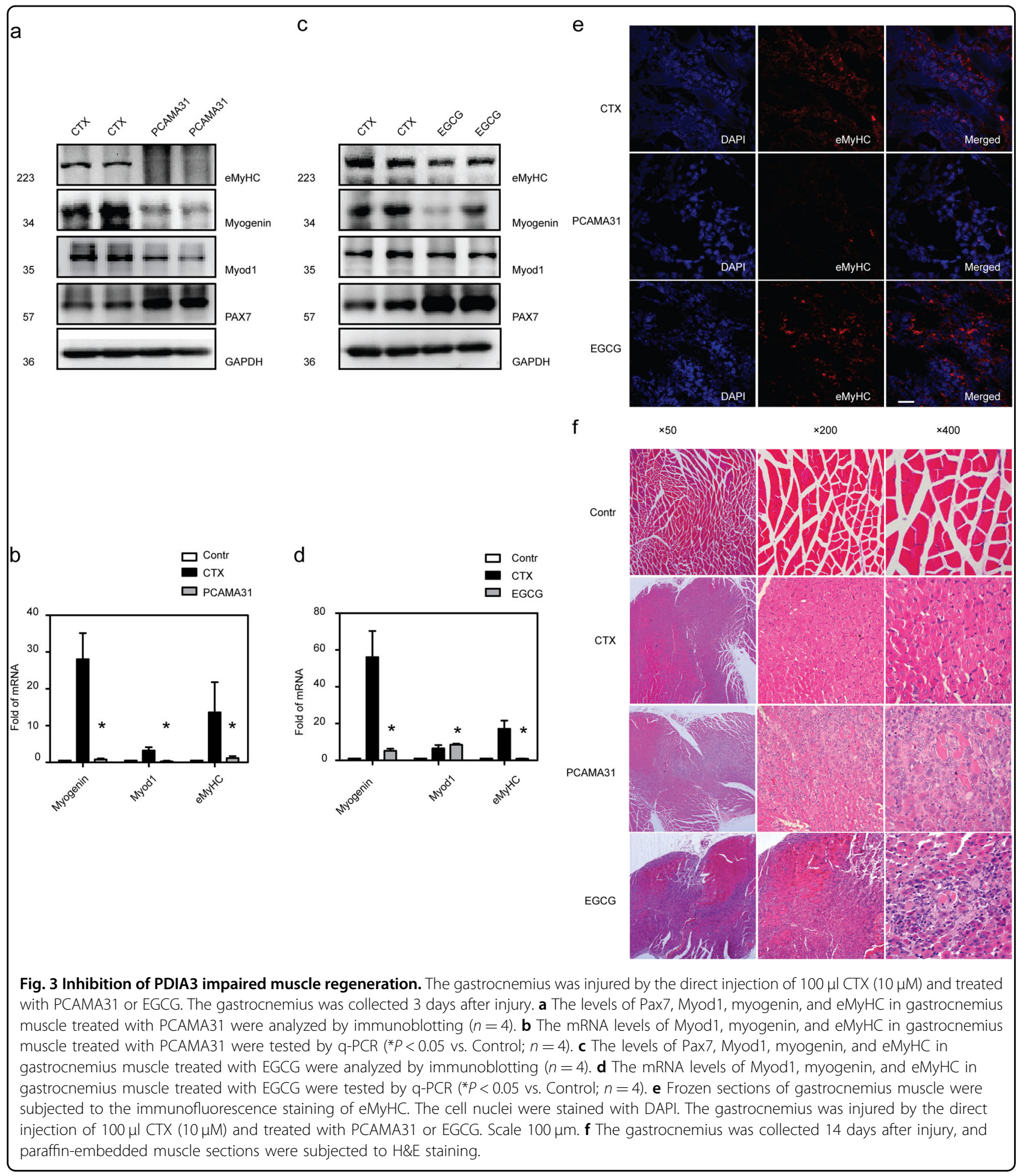

inhibitors in $\mathrm{C} 2 \mathrm{C} 12$ myoblasts. We initially examined the effect of PCAMA31 on myogenic marker expression and the fusion of $\mathrm{C} 2 \mathrm{C} 12$ myoblasts. As shown in Figs. 4b, c and 5, PCAMA31 significantly decreased Myod1, myogenin, and $\mathrm{MyHC}$ expression and inhibited the fusion of myoblasts. In the myoblast differentiation experiment, when treated with PDI (16F16), which is a widely used PDI inhibitor, myoblasts failed to express Myod1, myogenin, and $\mathrm{MyHC}$ or to fuse into myotubes (Figs. 4d, e and 5). To confirm the involvement of PDIA3 in myogenesis, we tested the myogenic expression and fusion of $\mathrm{C} 2 \mathrm{C} 12$ myoblasts by inhibiting PDIA3 using $\mathrm{EGCG}^{24}$. The results 


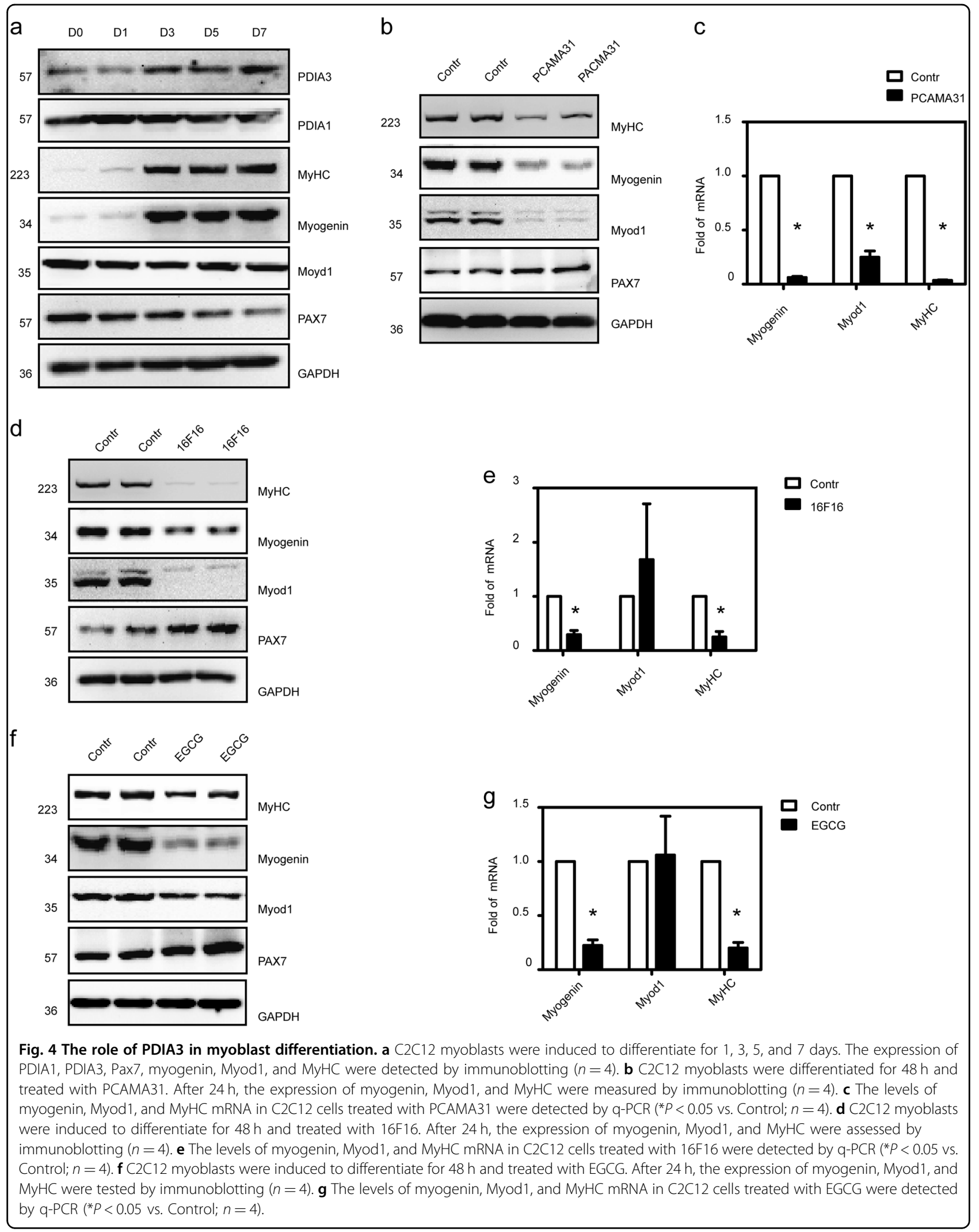




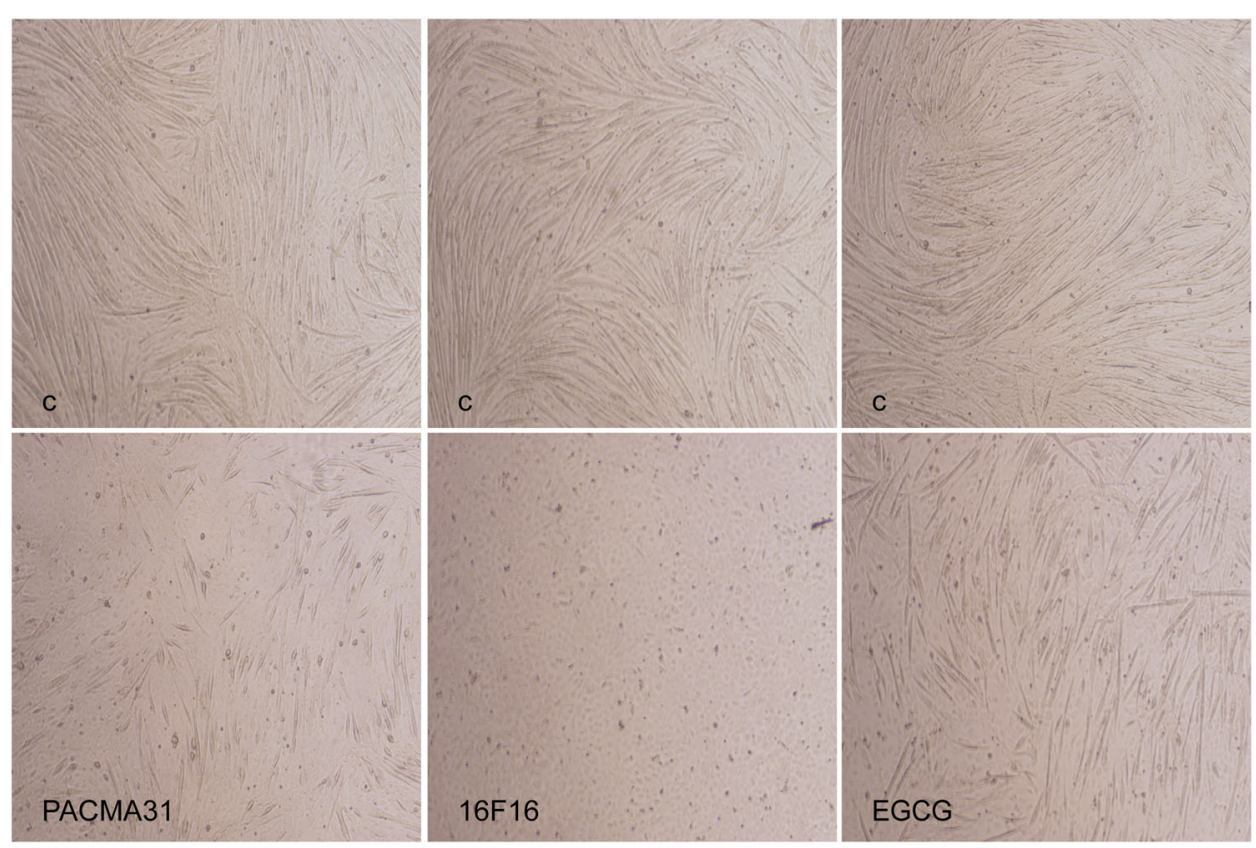

Fig. 5 The role of PDIA3 in myoblast fusion. C2C12 myoblasts were induced to differentiate for $48 \mathrm{~h}$ and were treated with PCAMA31, 16F16, and EGCG. After $72 \mathrm{~h}$, myotube formation was observed by a microscope. Scale $200 \mu \mathrm{m}$.

in Figs. 4f, $g$ and 5 show that EGCG had no influence on Myod1 expression but decreased myogenin and MyHC expression and inhibited the fusion of myoblasts. These results indicate that a wide range of PDI inhibitors can interfere with the capacity for myogenesis, and suggest that PDIA3 is a major PDI isoform that mediates the capacity for myogenesis.

Taken together, the results of these experiments show that the PDI isoform PDIA3 plays a major role in mediating myogenesis, whereas the PDI isoform PDIA1 plays little or no role in this process.

\section{Extracellular PDIA3 is crucial for myoblast differentiation and fusion}

To investigate whether extracellular PDIA3 is involved in myoblast differentiation and fusion, we first analyzed the effects of the membrane impermeable inhibitor bacitracin on myogenic marker expression and the fusion of myoblasts. When $\mathrm{C} 2 \mathrm{C} 12$ myoblasts were induced to differentiate in the presence of bacitracin, there were significant reductions in the amounts of the proteins Myod1, myogenin, and $\mathrm{MyHC}$, and the numbers of myotubes with respect to those in control cells (Fig. 6a, b, e). In addition to bacitracin, we performed $\mathrm{C} 2 \mathrm{C} 12$ differentiation in the presence of a blocking monoclonal anti-PDIA3 antibody. We found that the anti-PDIA3 antibody significantly decreased the number of myotubes and inhibited myogenin and $\mathrm{MyHC}$ expression but did not alter Myod1 expression (Fig. 6c-e).

Because the evidence from the inhibition studies suggested that there was a critical effect of extracellular PDIA3 activity on myoblast differentiation, we used immunofluorescence staining analysis to detect the presence of PDIA3 on myoblasts and myotube membrane surfaces. Live cell staining showed that PDIA3 was expressed on differentiated myotube membrane surfaces but not on undifferentiated myoblast membranes (Fig. 6f). Additionally, live cell staining showed that PDIA3 was co-localized on myotube membranes with the membrane protein marker MG53 (Fig. 6g). We further tested the expression of PDIA3 on membranes by immunoblotting. As Fig. 6h illustrates, PDIA3 was upregulated on differentiated myotube membranes compared with undifferentiated myoblast membranes. In addition, we used an ELISA assay to measure the level of PDIA3 protein in the extracellular medium of $\mathrm{C} 2 \mathrm{C} 12$ cells before differentiation and after differentiation. PDIA3 protein was present in the extracellular medium, and a dramatic increase in this protein was observed at 1 and 2 days after differentiation (Fig. 6i).

Thus, these results indicate that PDIA3 is secreted during $\mathrm{C} 2 \mathrm{C} 12$ differentiation, and this autocrine signaling by PDIA3 is crucial to myoblast differentiation and fusion.

\section{Extracellular PDIA3 regulates myoblast differentiation and fusion via AKT/mTOR signaling}

To assess the time-course of AKT/mTOR pathway activity in the CTX injury model, we first examined the phosphorylation levels of AKT (p-AKT) in muscle after injury. Accompanied by the induction of $\mathrm{MyHC}$ expression, the level of p-AKT was significantly increased in regenerated myofibers (Fig. 7a). In vitro, the levels of 


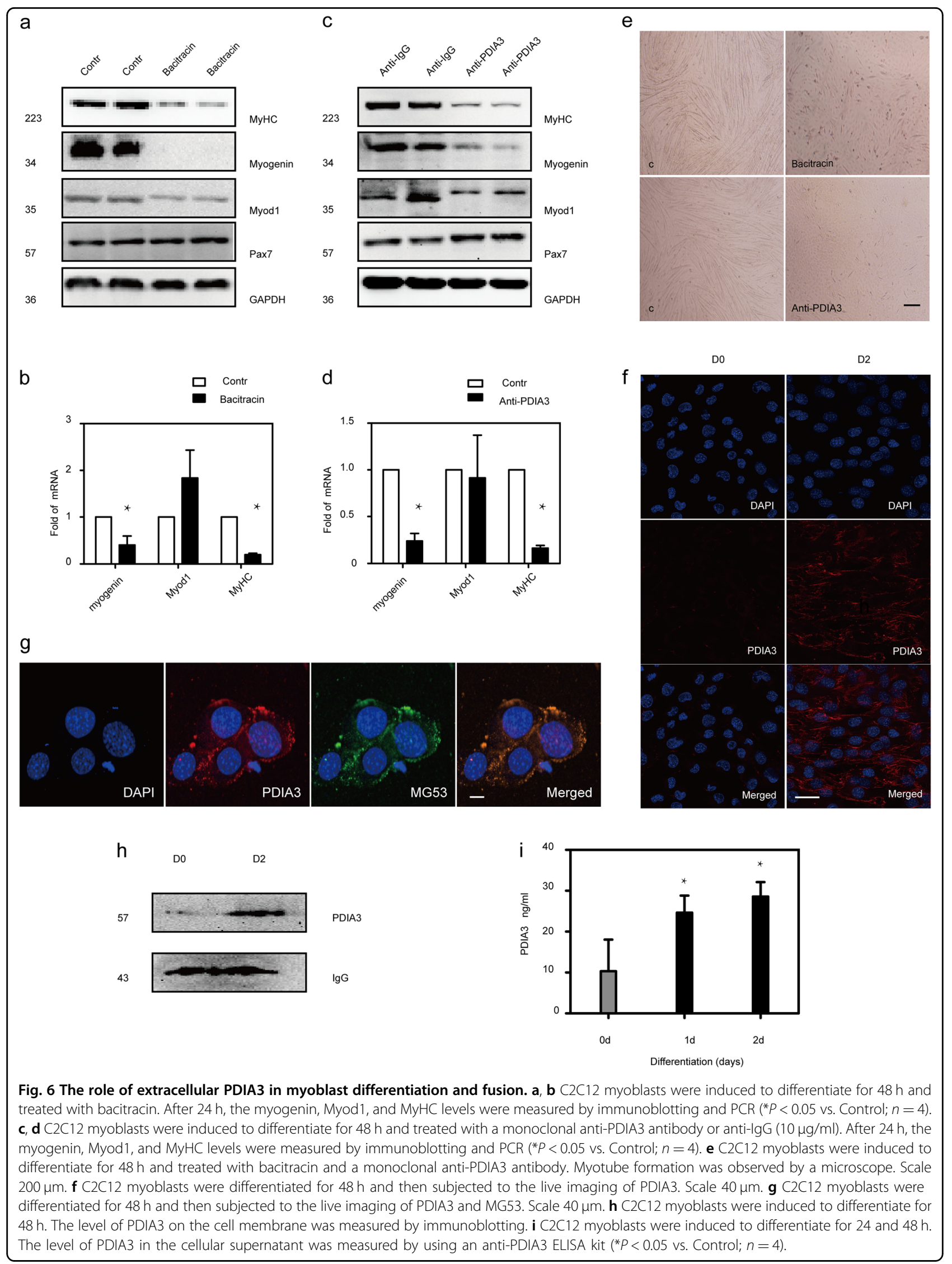


a
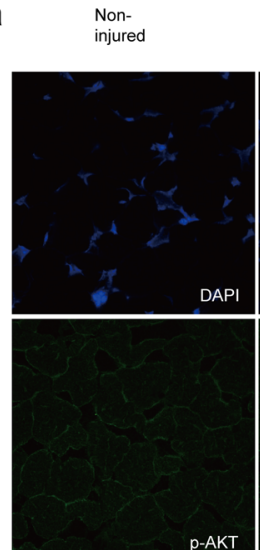

DAPI

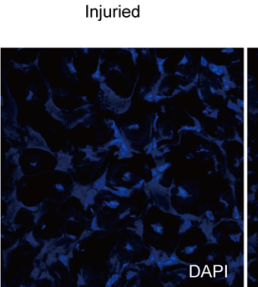

EGCG

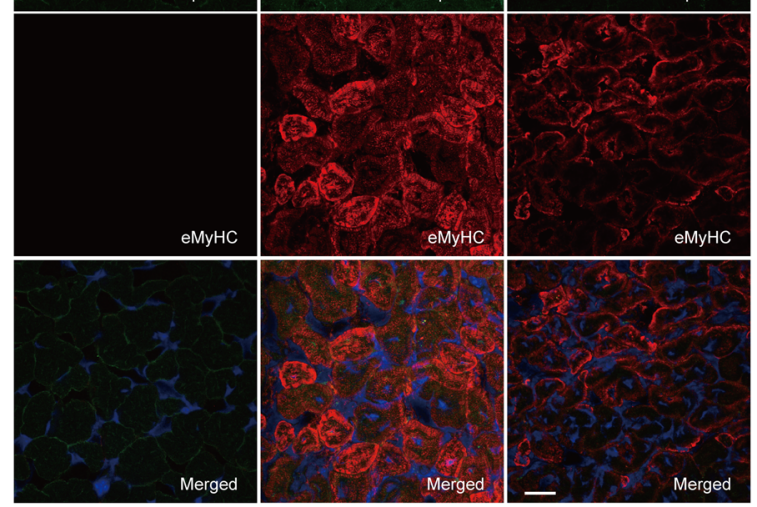

d

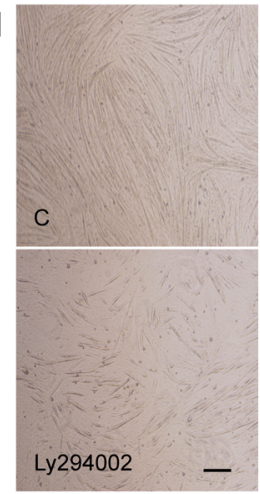

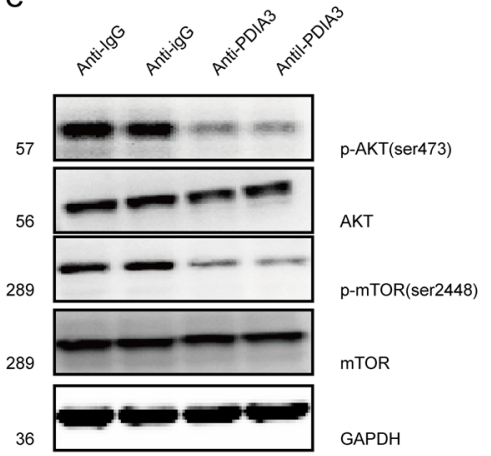

$\mathrm{b}$

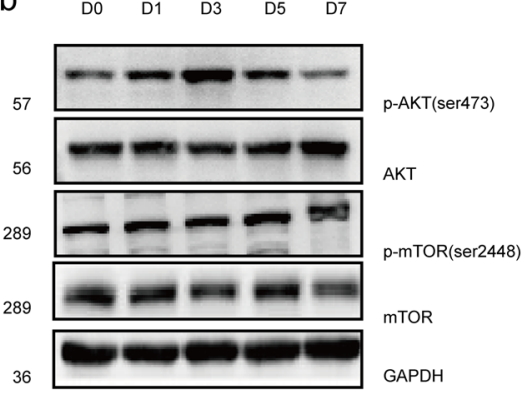

C

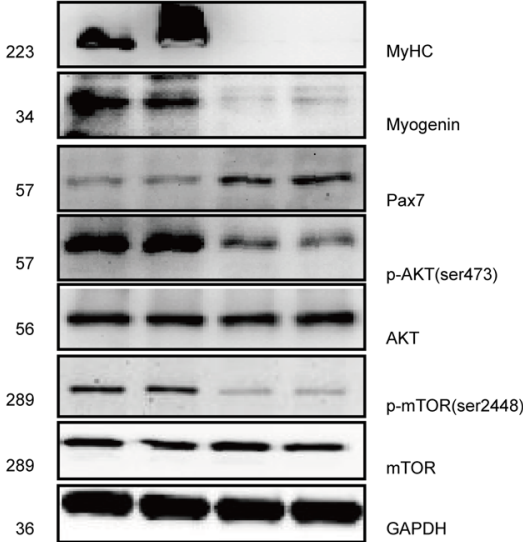

f

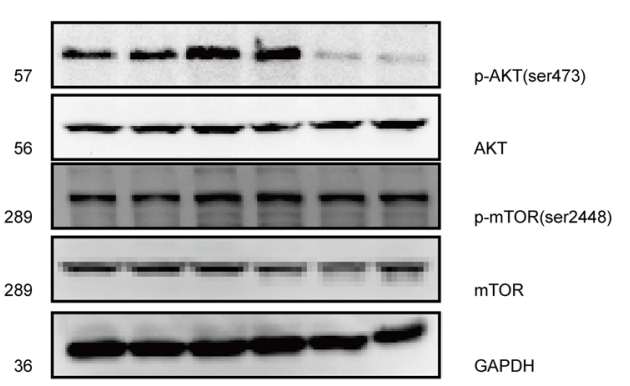

Fig. 7 PDIA3 regulates myoblast differentiation through AKT/mTOR signaling. a The gastrocnemius in mice was injured by the direct injection of $100 \mu \mathrm{l} \mathrm{CTX}(10 \mu \mathrm{M})$ and the intraperitoneal injection of EGCG. On day 7 post injury, the gastrocnemius was harvested from euthanized mice. Frozen sections of gastrocnemius muscle were subjected to the immunofluorescence staining of p-AKT and eMyHC. Scale $20 \mu m$. b C2C12 myoblasts were induced to differentiate for 1, 3, 5, and 7 days. The levels of the p-AKT, AKT, p-mTOR, and mTOR proteins were assessed by immunoblotting $(n=4)$. $\mathbf{c}$ C2C12 myoblasts were induced to differentiate for $48 \mathrm{~h}$ and treated with LY90042. After $24 \mathrm{~h}$, the levels of the MyHC, myogenin, p-AKT, AKT, p-mTOR, and mTOR proteins were assessed by immunoblotting $(n=4)$. $\mathbf{d}$ C2C12 myoblasts were induced to differentiate for $48 \mathrm{~h}$ and treated with LY90042. After $72 \mathrm{~h}$, myotube formation was observed by a microscope. Scale $200 \mu \mathrm{m}$. e C2C12 myoblasts were induced to differentiate for $48 \mathrm{~h}$, followed by treatment with an anti-PDIA3 antibody for $24 \mathrm{~h}$. The expression of p-AKT, AKT, p-mTOR, and mTOR were measured by immunoblotting $(n=4)$. $\mathbf{f}$ C2C12 myoblasts were induced to differentiate for $48 \mathrm{~h}$ and treated with IGF-1 $(2 \mu \mathrm{g} / \mathrm{ml})$ for $24 \mathrm{~h}$ in the absence or presence of anti-PDIA3 $(10 \mu \mathrm{g} / \mathrm{ml})$ antibody. The levels of the p-AKT, AKT, p-mTOR, and mTOR proteins were measured by immunoblotting $(n=4)$.

P-AKT and P-mTOR were both increased during $\mathrm{C} 2 \mathrm{C} 12$ myoblast differentiation, while the total AKT and mTOR levels were not altered (Fig. 7b). Moreover, we induced $\mathrm{C} 2 \mathrm{C} 12$ myoblast differentiation in the presence of
LY294002, a compound that inhibits AKT/mTOR signaling. As shown in Fig. 7c, d, LY294002 significantly decreased the expression of $\mathrm{p}-\mathrm{AKT}, \mathrm{p}-\mathrm{mTOR}$, myogenin, and $\mathrm{MyHC}$ and blocked myoblast fusion. These results 
confirm that AKT/mTOR signaling is critical for myogenesis and muscle regeneration.

To determine whether PDIA3 regulates myoblast differentiation and fusion via AKT/mTOR signaling, we first showed that the inhibition of PDIA3 significantly reduced p-AKT and eMyHC expression in CTX-damaged muscle (Fig. 7a). In vitro, p-AKT and p-mTOR expression were also inhibited by anti-PDIA3 antibody during myoblast differentiation (Fig. 7e). IGF-1, an essential factor in myoblast differentiation, clearly increased the levels of $\mathrm{p}$ AKT and p-mTOR; however, these effects were diminished by the anti-PDIA3 antibody (Fig. 7f).

\section{PDIA3-mediated myoblast differentiation and fusion depends on $\beta 3$ integrin}

To test whether PDIA3 mediation of myogenesis depended on $\beta 3$ integrin, we first detected the expression of $\beta 3$ integrin in regenerated muscles using the CTX injury model. Immunofluorescence staining revealed that $\beta 3$ integrin expression was induced in regenerated myofibers with centralized nuclei (Fig. 8a). Similar to PDIA3, $\beta 3$ integrin was undetected in uninjured muscles but was expressed in injured muscle at 3-7 days after injury (Fig. $8 b)$. An in vitro study showed that $\beta 3$ integrin was upregulated during myogenic differentiation (Fig. 8c). We further induced myoblast differentiation by blocking $\beta 3$ integrin using a monoclonal anti- $\beta 3$ integrin antibody. As Fig. $8 d$, e show, the anti- $\beta 3$ integrin antibody significantly decreased p-AKT, P-mTOR, myogenin, and MyHC expression, ultimately inhibiting myoblast fusion. These results indicate that $\beta 3$ integrin is a major component that facilitates myogenesis and muscle regeneration through the AKT/mTOR pathway.

Because the evidence obtained from the immunofluorescence staining suggested that PDIA3 colocalized with $\beta 3$ integrin in the muscle membrane in neonatal mice (Fig. $8 \mathrm{a}$ ), we performed immunoprecipitation assays using lysates derived from differentiated myoblasts. We found that PDIA3 was coimmunoprecipitated with $\beta 3$ integrin (Fig. 8f). We further carried out myoblast differentiation by activating $\beta 3$ integrin using recombinant vitronectin, which is a widely used ligand of $\beta 3$ integrin. Vitronectin treatment significantly enhanced myogenin and $\mathrm{MyHC}$ expression; however, these effects were diminished by the anti-PDIA3 antibody (Fig. 8g). This observation suggested that PDIA3 regulated $\beta 3$ integrin-mediated myoblast differentiation via the AKT/mTOR-signaling pathway.

\section{Discussion}

The current study demonstrates that extracellular PDIA3 plays a critical role in regulating myoblast differentiation and fusion during muscle regeneration. Furthermore, our results reveal that extracellular PDIA3 is associated with activated $\beta 3$ integrin and AKT/mTOR signaling in myogenesis during muscle regeneration. Thus, we report the regulatory role of PDIA3 in muscle regeneration, which is mainly mediated by $\beta 3$ integrin, in myoblast differentiation and fusion via $\mathrm{AKT} / \mathrm{mTOR}$ signaling.

During muscle regeneration, myogenesis is tightly regulated by a combination of signaling molecules that successively induces the expression of myogenic transcription factors (such as Pax7, Myod1, and myogenin) to control SC self-renewal and differentiation ${ }^{25}$. We demonstrated here that PDIA3 expression was induced in mature regenerated myofibers (myogenin-positive and MyHC-positive) but was undetected in activated SCs (Pax7-positive and Myod1positive), indicating a physiological role of PDIA3 in terminal myogenic differentiation rather than the earlier initiation of myogenesis. The in vivo studies further revealed that the inhibition of PDIA3 by EGCG could suppress myoblast terminal differentiation and fusion. However, we still need to consider the potential effects of EGCG on the activity of members of the PDI family, such as PDIA1 and PDIA224.

Interestingly, we have shown that PDIA3 accumulates on the membrane in regenerated muscle, which is also detected outside the cell during myoblast differentiation in vitro. The specific inhibition of extracellular PDIA3 activity with monoclonal anti-PDIA3 antibody significantly inhibits myogenic terminal differentiation and the fusion of myoblasts. Thus, it appears that extracellular PDIA3 is required for myogenic terminal differentiation and the fusion of myoblasts in the late phase of muscle regeneration.

Although a previous study reported that extracellular PDIA3 could directly bind to $\beta 3$ integrin to regulate $\alpha v \beta 3$ integrin-mediated coagulation, neutrophil migration, $\mathrm{T}$ cell migration, and sperm-egg fusion ${ }^{19-23,26}$, it was unknown whether this PDIA3-mediated $\beta 3$ integrin activity was involved in myogenesis during muscle regeneration. Recently, $\beta 3$ integrin has been reported to be transiently induced in activated SCs, which is essential for the initiation of myogenesis in SCs, and its deficiency disrupts myogenesis and impairs muscle regeneration ${ }^{17}$. In this study, we found that $\beta 3$ integrin expression is also induced in regenerating myofibers and gradually increases in $\mathrm{C} 2 \mathrm{C} 12$ myoblasts during differentiation and fusion. The administration of an anti- $\beta 3$ integrin antibody significantly inhibited myogenic terminal differentiation and impaired myotube formation, suggesting that $\beta 3$ integrin is also required for these two main steps of myogenesis. Furthermore, we demonstrated that PDIA3 directly bound to $\beta 3$ integrin and that $\beta 3$ integrin co-localized with PDIA3 in myofiber membranes. The positive effects of $\beta 3$ integrin-mediated myoblast differentiation was diminished by the anti-PDIA3 antibody. Thus, we concluded that extracellular PDIA3 regulates $\beta 3$ integrinmediated myoblast differentiation and fusion.

To the best of our knowledge, the AKT/mTOR-associated signaling pathway is crucial for myogenic terminal 
a
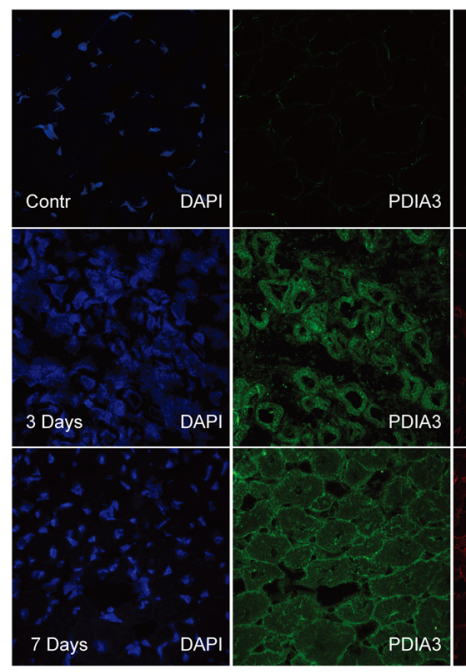

d

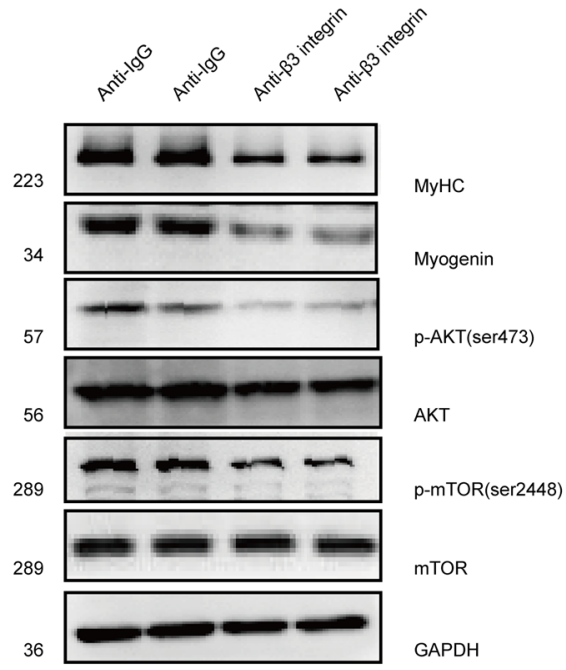

f

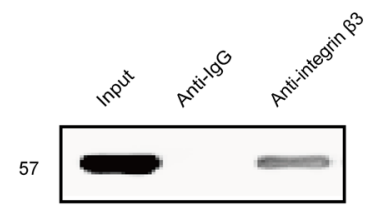

PDIA3 b

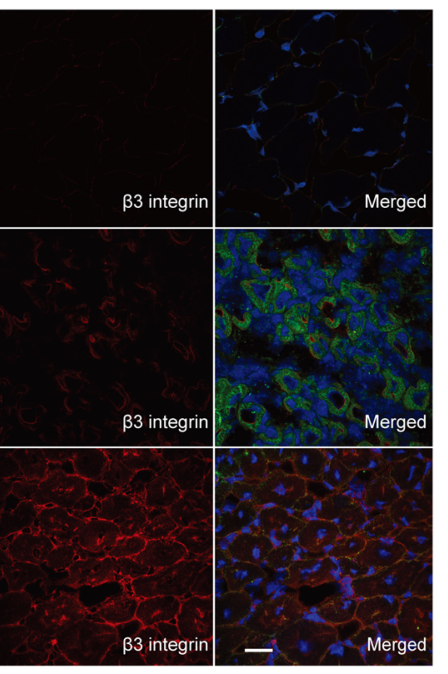

110

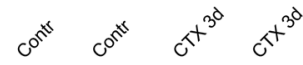

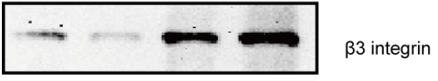

36

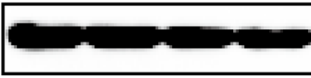

GAPDH

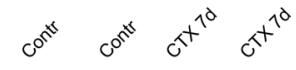

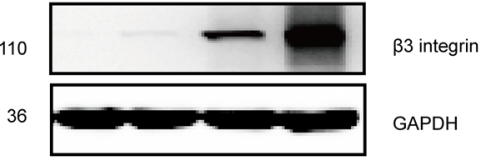

C

110

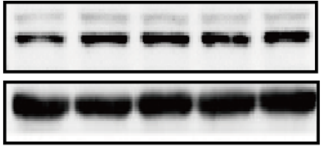

$\beta 3$ integrin

GAPDH e

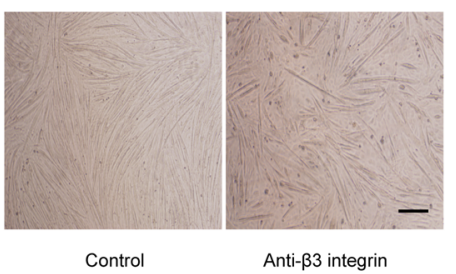

g

223

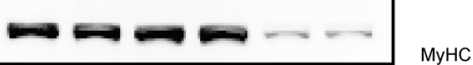

34
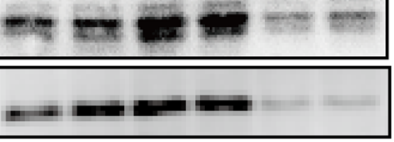

Myogenin

p-AKT(ser473)

56

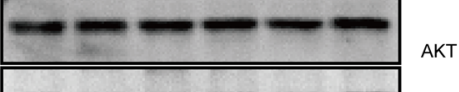

289

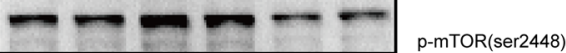

289

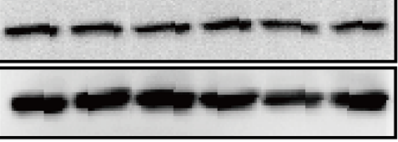

MTOR

GAPDH

Fig. 8 PDIA3 regulates $\beta 3$ integrin-mediated myoblast differentiation and fusion. a Frozen sections of gastrocnemius muscle were subjected to the immunofluorescence costaining of $\beta 3$ integrin and PDIA3. Scale $20 \mu \mathrm{m}$. $\mathbf{b}$ The gastrocnemius in mice were injured by the direct injection of $100 \mu \mathrm{l} \mathrm{CTX}(10 \mu \mathrm{M})$ and collected at 3 and 7 days after injury. The level of $\beta 3$ integrin protein was measured by immunoblotting. $(n=4)$. c C2C12 myoblasts were subjected to differentiation for $1,3,5$, and 7 days. The $\beta 3$ integrin levels were measured by immunoblotting $(n=4)$. $\mathbf{d} C 2 \mathrm{C} 12$ myoblasts were induced to differentiate for $48 \mathrm{~h}$, followed by monoclonal anti- $\beta 3$ integrin $(10 \mu \mathrm{g} / \mathrm{ml})$ treatment for $24 \mathrm{~h}$. The levels of p-AKT, AKT, pmTOR, mTOR, myogenin, and MyHC were measured by immunoblotting $(n=4)$. e C2C12 myoblasts were induced to differentiate for $48 \mathrm{~h}$, followed by monoclonal anti- $\beta 3$ integrin $(10 \mu \mathrm{g} / \mathrm{ml})$ treatment for $72 \mathrm{~h}$. Myotube formation was observed by a microscope. Scale $200 \mu \mathrm{m}$. f The gastrocnemius in mice were injured by the direct injection of $100 \mu \mathrm{l} C T X(10 \mu \mathrm{M})$ and collected at 7 day after injury. The muscle lysates were subjected to immunoprecipitation with an anti-PDIA3 antibody and were immunoblotted with anti- $\beta 3$ integrin antibody $(n=4)$. g C2C12 myoblasts were induced to differentiate for $48 \mathrm{~h}$ and incubated with recombinant human vitronectin $(2 \mu \mathrm{g} / \mathrm{ml})$ for $24 \mathrm{~h}$ in the absence or presence of an anti-PDIA3 $(10 \mu \mathrm{g} / \mathrm{ml})$ antibody. The levels of $\mathrm{p}-\mathrm{AKT}, \mathrm{AKT}, \mathrm{p}-\mathrm{mTOR}$, mTOR, myogenin, and MyHC were measured by immunoblotting $(n=4)$. 
differentiation and remodeling during muscle regeneration $^{27}$. It has been established that muscle injury induces the expression of IGF-1 and further stimulates the AKT/ mTOR-signaling pathway, which is beneficial for muscle regeneration ${ }^{12,28}$. Here, we observed the significant activation of $\mathrm{AKT} / \mathrm{mTOR}$ signaling in regenerating myofibers. The blockade of myogenin and $\mathrm{MyHC}$ expression and myoblast fusion by LY294002 further supported the notion that $\mathrm{AKT} / \mathrm{mTOR}$ signaling plays a key role in terminal myogenic differentiation and fusion. We further found that cell-surface PDIA 3 and $\beta 3$ integrin were two components involved in maintaining $\mathrm{AKT} / \mathrm{mTOR}$ signaling during muscle regeneration. In addition, the effect of $\beta 3$ integrin on AKT/mTOR signaling was diminished by the anti-PDIA3 antibody, indicating that $\beta 3$ integrinmediated AKT/mTOR signaling during myogenesis depends on extracellular PDIA3.

In summary, our findings have identified PDIA3 as an autocrine factor expressed specifically in regenerating myofibers that is required for the activation of $\beta 3$ integrin and $\mathrm{AKT} / \mathrm{mTOR}$ signaling during myoblast differentiation and fusion. However, considering the complexity and multifunctional activity of members of the PDI family, further study of the mechanism underlying the effects of other PDIs (such as PDIA1 and PDIA2) on myogenesis is warranted.

\section{Acknowledgements \\ This work was supported by the Natural Science Foundation of China (Nos. 81571899; 81701903) and the International Cooperation Program of TMMU (SWH2O16HWHZ03). The funder did not participate in designing this study, collecting and analyzing data, preparing the manuscript or the decision to publish.}

\section{Author details \\ 'State Key Laboratory of Trauma, Burns and Combined Injury, Clinical Medical Research Center, Southwest Hospital, Third Military Medical University (The Army Medical University), Chongqing 400038, China. ${ }^{2}$ Department of Burns and Plastic Surgery, Chengdu Second People's Hospital, Chengdu 610011, China. ${ }^{3}$ Department of Burns, Union Hospital, Fujian Medical University, Fuzhou 350001, China. ${ }^{4}$ Department of Geriatric Medicine, Chengdu Second People's Hospital, Chengdu 610011, China. ${ }^{5}$ Shriners Burns Hospital, Massachusetts General Hospital, Harvard Medical School, Boston, MA 02114, USA}

\section{Author contributions}

X.P. and C.W. designed the experiments. Y.Z. and C.W. carried out all vivo and vitro experiments and were involved in the data collection. D.W. and Z.W. performed the optical and confocal microscopy. G.Y. and Y.S. were responsible for the image analysis. C.W. analyzed the data and drafted the manuscript. X.L.X., Y.M.Y. and X.P. supervised the experimental procedures and revised the manuscript.

\section{Conflict of interest}

The authors declare that they have no conflict of interest.

\section{Publisher's note}

Springer Nature remains neutral with regard to jurisdictional claims in published maps and institutional affiliations.

Received: 17 December 2018 Revised: 8 October 2019 Accepted: 11 October 2019.

Published online: 20 January 2020

\section{References}

1. Kjobsted, R. et al. AMPK in skeletal muscle function and metabolism. FASEB J. 35, 23-34 (2017).

2. Giudice, J. \& Taylor, J. M. Muscle as a paracrine and endocrine organ. Curr. Opin. Pharmacol. 34, 49-55 (2017).

3. Spriet, L. L. \& Whitfield, J. Taurine and skeletal muscle function. Curr. Opin. Clin. Nutr. Metab. Care 18, 96-101 (2015).

4. Wang, C. et al. A novel perspective for burn-induced myopathy: membrane repair defect. Sci. Rep. 6, 31409 (2016)

5. Shi, H., Xie, H., Zhao, Y., Lin, C. \& Cui, F. Myoprotective effects of bFGF on skeletal muscle injury in pressure-related deep tissue injury in rat. Burns Trauma. 17, 26 (2016).

6. Hurtgen, B. et al. Autologous minced muscle grafts improve endogenous fracture healing and muscle strength after musculoskeletal trauma. Physiol. Rep. 14, e13362 (2017). https://doi.org/10.14814/phy2.13362.

7. Guiraud, S. \& Davies, K. E. Pharmacological advances for treatment in Duchenne muscular dystrophy. Curr. Opin. Pharmacol. 34, 36-48 (2017).

8. Kwee, B. J. \& Mooney, D. J. Biomaterials for skeletal muscle tissue engineering. Curr. Opin. Biotechnol. 47, 16-22 (2017).

9. Cesana, M. et al. A long noncoding RNA controls muscle differentiation by functioning as a competing endogenous RNA. Cell 147, 358-369 (2011).

10. Fu, X., Zhu, M. J., Dodson, M. V. \& Du, M. AMP-activated protein kinase stimulates Warburg-like glycolysis and activation of satellite cells during muscle regeneration. J. Biol. Chem. 290, 26445-26456 (2015).

11. Garcia-Prat, L. et al. Autophagy maintains stemness by preventing senescence. Nature 529, 37-42 (2016).

12. Demonbreun, A. R. \& McNally, E. M. Muscle cell communication in development and repair. Curr. Opin. Pharmacol. 34, 7-14 (2017).

13. Takemoto, Y. \& Fukada, S. I. Molecular mechanism maintaining muscle satellite cells and the roles in sarcopenia. Clin. Calcium 27, 339-344 (2017).

14. Giancotti, F. G. \& Ruoslahti, E. Integrin signaling. Science 285, 1028-1032 (1999).

15. Carpenter, B. et al. Integrin alpha6beta4 promotes autocrine epidermal growth factor receptor (EGFR) signaling to stimulate migration and invasion toward hepatocyte growth factor (HGF). J. Biol. Chem. 290, 27228-27238 (2015).

16. Saegusa, J. et al. The direct binding of insulin-like growth factor-1 (IGF-1) to integrin alphavbeta3 is involved in IGF-1 signaling. J. Biol. Chem. 284, 24106-24114 (2009).

17. Takada, Y., Takada, Y. K. \& Fujita, M. Crosstalk between insulin-like growth factor (IGF) receptor and integrins through direct integrin binding to IGF1. Cytokine Growth Factor Rev. 34, 67-72 (2017).

18. Liu, H., Niu, A., Chen, S. E. \& Li, Y. P. Beta3-integrin mediates satellite cell differentiation in regenerating mouse muscle. FASEB J. 25, 1914-1921 (2011).

19. Xu, S., Sankar, S. \& Neamati, N. Protein disulfide isomerase: a promising target for cancer therapy. Drug Discov. Today 19, 222-240 (2014).

20. Bowley, S. R., Fang, C., Merrill-Skoloff, G., Furie, B. C. \& Furie, B. Protein disulfide isomerase secretion following vascular injury initiates a regulatory pathway for thrombus formation. Nat. Commun. 8, 14151 (2017).

21. Hahm, E. et al. Extracellular protein disulfide isomerase regulates ligandbinding activity of alphaMbeta2 integrin and neutrophil recruitment during vascular inflammation. Blood 121, 3789-3800 (2013). s3781-3715.

22. Bi, S., Hong, P. W., Lee, B. \& Baum, L. G. Galectin-9 binding to cell surface protein disulfide isomerase regulates the redox environment to enhance T-cell migration and HIV entry. Proc. Natl Acad. Sci. USA 108, 10650-10655 (2011).

23. Ellerman, D. A., Myles, D. G. \& Primakoff, P. A role for sperm surface protein disulfide isomerase activity in gamete fusion: evidence for the participation of ERp57. Dev. Cell 10, 831-837 (2006).

24. Trnkov, L. et al. Green tea catechins can bind and modify ERp57/PDIA3 activity. Biochim. Biophys. Acta 1830, 2671-2682 (2013).

25. Baghdadi, M. B. \& Tajbakhsh, S. Regulation and phylogeny of skeletal muscle regeneration. Dev. Biol. 433, 200-209 (2018).

26. Mor-Cohen, R. et al. Disulfide bonds as regulators of integrin function in thrombosis and hemostasis. Antioxid. Redox Signal. 24, 16-31 (2016).

27. Zeng, Q. et al. Protective effects of sonic Hedgehog against ischemia/reperfusion injury in mouse skeletal muscle via AKT/mTOR/p70S6K signaling. Cell. Physiol. Biochem. 43, 1813-1828 (2017).

28. Witt, R. et al. Mesenchymal stem cells and myoblast differentiation under HGF and IGF-1 stimulation for 3D skeletal muscle tissue engineering. BMC Cell Biol. 18, 15 (2017). 